\title{
Derechos sociales y políticas sociales: en busca de la estabilidad y el equilibrio en América Latina
}

\section{Social Rights and Social Policies: in search of Stability and the balance in Latin America}

\section{Direitos sociais e políticas sociais: em busca de estabilidade e o equilíbrio na América Latina}

\author{
Fernando Montero-Cordero \\ Vicerrectoría de Extensión \\ Universidad Nacional \\ Recibido: 2/2/2015 Aceptado: 5/5/2015 \\ http://dx.doi.org/10.15359/tdna.31-58.4
}

\section{Resumen}

Más allá de lo que pensamos y conocemos del Estado y sus funciones, las políticas sociales han sido pilar fundamental para el combate contra la pobreza y la desigualdad, en mayor o menor medida, según las condiciones que presenten las economías. Los derechos sociales otorgados a través del Estado de derecho, han permitido que países en desarrollo transformen su estructura socioeconómica en igualdad y bienestar común.

La ocupación, la gerencia social, la estabilidad

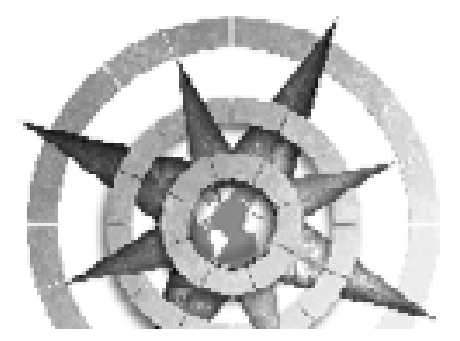

macroeconómica y la heterogeneidad estructural son factores comunes en el análisis y aplicación de las acciones propias del Estado. La combinación en el análisis de dichos factores nos da como resultado una gama de posibilidades de propuestas entorno al desarrollo y mejoramiento de la calidad de vida.

Palabras clave: Estado de Derecho, desarrollo, calidad de vida, políticas sociales, América Latina, Costa Rica

\section{Abstract}

Beyond what we think and know about the State and its functions, social policies have been a cornerstone for the fight against poverty and inequality to a greater or lesser degree depending on the economy conditions. 
Social rights granted by the rule of law have allowed developing countries to transform their socioeconomic structure in equality and common welfare. Occupation, social management, macroeconomic stability and structural heterogeneity are common factors in the analysis and implementation of the State's own actions. The combination in the analysis of such factors gives, as a result a range of proposals for the development and the improving the quality of life.

Keywords: Rule of law, development, quality of life, social policies, Latin America, Costa Rica

\section{Resumo}

As políticas sociais têm sido um elemento fundamental para a luta contra a pobreza ea desigualdade em graus variados de acordo com as condições apresentadas economias. Os direitos sociais concedidos pelo Estado de direito têm permitido os países em desenvolvimento a transformar a sua estrutura socioeconômica em igualdade e bem-estar comum. Ocupação, gestão social, a estabilidade macroeconómica ea heterogeneidade estrutural são fatores comuns na análise e implementação de acções próprias do Estado. A combinação da análise desses fatores resulta em uma gama de possibilidades para propostas sobre o desenvolvimento e uma melhor qualidade de vida.
Palavras chave: Estado de direito, desenvolvimento, qualidade de vida, a política social, a América Latina, Costa Rica

\section{Las políticas sociales en un contex- to de desigualdad}

La característica fundamental del bien común es el tratamiento que se da a lo social en un contexto donde las economías tratan de acortar las diferencias entre ricos y pobres.

La agenda política siempre está plagada de planes de desarrollo que permitan cubrir las necesidades de la población y asegurar la dignidad humana de sus integrantes. Estrechar esa brecha entre los que tienen demasiado y los que nada tienen, es el fundamento de la política social, cuyos recursos se nutren comúnmente por las cargas impositivas, lo que permite la redistribución del ingreso, al pagar más los que más tienen, llamado comúnmente como políticas progresivas.

Pero ¿qué es una política social y de qué se ocupa? Es una rama de la política que se ocupa de detectar los problemas sociales que derivan en pobreza y marginación (falta de trabajo, vivienda digna, educación, alimentos) para buscar los recursos y medios técnicos que les den solución, pues su objetivo es el bienestar general de la población.

76 Derechos sociales y políticas sociales: en busca de la estabilidad y el equilibrio en América Latina Fernando Montero-Cordero 
Por tanto podríamos decir que es la intervención del Estado en la sociedad, como planteamiento reactivo, que busca la protección social frente a los efectos de la desigualdad social y los desequilibrios sociales, se enfoca a la resolución de los denominados problemas sociales o asuntos sociales. Como ciencia es un área de conocimiento interdisciplinar y aplicada que tiene como fin el análisis de las transformaciones del Estado del bienestar y las respuestas sociales a las necesidades, retos y riesgos sociales.

Sin lugar a duda la política social tiende a examinar los aspectos de la economía, la sociedad, la población y la política que son necesarios para la existencia social digna y los medios para conseguirla; el Estado como encargado de la política social diseña programas de intervención social que permitan evitar la pobreza y la exclusión social, reducir las desigualdades sociales, aumentar la formación orientada a la participación de los individuos en igualdad de condiciones en la sociedad (participación social) y mejorar la eficiencia y la calidad de vida.

No todos los países latinoamericanos han podido mantener condiciones de política social satisfactoria, reflejada en los niveles de pobreza, empleo y riqueza:
Costa Rica y Uruguay (que, como ya he dicho anteriormente, son los únicos países de América Latina donde, junto con los derechos políticos, los derechos civiles y la rendición de cuentas horizontal son razonablemente efectivos) sugieren que una de las direcciones de causalidad va de estos derechos a la estructura social (O’Donnell, 2001: p. 15)

El Estado de derecho es fundamental para poner en práctica las políticas sociales que permiten generar las formas y estado que el ciudadano requiere en concubinato con las leyes y principios del desarrollo con equidad:

el estado de derecho debería considerarse la norma basada en la legalidad de un estado democrático.... establece redes de responsabilidad y rendición de cuentas que comportan que todos los agentes, privados y públicos, incluyendo los cargos más altos del régimen, estén sujetos a controles apropiados y legalmente establecidos sobre la legalidad de sus actos (O’Donnell, 2001, p. 1).

\section{La gerencia social... Estado públi- co-Estado privado}

¿Cómo puede el Estado llevar a cabo políticas sociales y garantizar los derechos sociales? Lo fundamental para la aplicación de sanas políticas 
sociales es incorporar gran parte de las técnicas y conceptos que han tenido éxito en el sector privado, pero que aún no han podido ser incorporadas en el sector público.

Es la modernización en la Gerencia Social la que permite garantizar un proceso que guie o dirija organizaciones o instituciones públicas hacia el logro de su misión y los objetivos que estas se han propuesto en políticas, programas y proyectos, en un ambiente complejo que requiere una negociación constante con actores, grupos internos y externos del Estado, haciendo uso eficiente y eficaz de los recursos.

El gerente público debe asumir una serie de roles y llevar a cabo una serie de intervenciones gerenciales que aseguren el cumplimiento de la misión, los objetivos y las metas organizacionales que implican el dominio tanto de funciones técnicas como políticas, donde el papel de la negociación como acto político que es, resulta fundamental para el desarrollo (Vargas, 2012).

La gerencia pública apunta a mejorar las condiciones sobre las cuales las políticas públicas sociales tengan un mayor impacto no solo en el crecimiento, sino también en el desarrollo:
La introducción del management en el Sector Social es una necesidad apremiante en que los Estados deben mantener un esfuerzo sostenido. Debemos aprovechar en dicho sector, las ventajas del nuevo paradigma gerencial que privilegia al ser humano, el trabajo sinergético en equipo y la polifuncionalidad, superando la feudocracia que estimuló el desarrollo del modelo burocrático (Vargas, 2012: p. 116)

Las políticas sociales asertivas deberían impactar positivamente en la disminución de la pobreza, aunque muchas veces esta pobreza es entendida de diversas formas. Para Carlos Sojo $^{3}$ la pobreza se presenta de muy distintas formas: "a la disposición de recursos tangibles o inmateriales: reconocimiento o discriminación, incluso el trato aparecen enunciados como elementos definitorios de la desigualdad". (Sojo, 2010: p. 120)

La pobreza y la desigualdad entendida como la posición que el individuo (en este caso costarricense) cree ocupar en la estructura social, específicamente en la jerarquización de las posiciones de clase asociadas a la riqueza. La confianza en la capacidad igualadora de las políticas públicas es un rasgo relevante de la

3 Entrevista de Carlos Sojo a inmigrante árabe

78 Derechos sociales y políticas sociales: en busca de la estabilidad y el equilibrio en América Latina

Fernando Montero-Cordero 
percepción de equidad y de justicia de la sociedad costarricense, aspecto que en mi opinión deriva de la valoración de una experiencia histórica de relación con un Estado vinculado al bienestar de las personas.

Según lo señalado, muchas economías, como la costarricense, han considerado que la igualación de las acciones públicas competen exclusivamente al Estado, como ente proveedor de todas las necesidades del individuo: "Todavía estamos con ese concepto de que todo lo queremos regalado, ve, si el tico es dado a ese tipo de errores. Una de las causas de esta pobreza es esto, en que dependemos muchas veces de lo que el gobierno nos va a regalar" (Sojo, p. 127).

Para Sojo, el costarricense ve al Estado como el responsable de la generación de políticas que favorecen a los que poseen menos riqueza y oportunidades:

Predomina una idea liberal de la desigualdad, fuertemente centrada en el plano socioeconómico que asigna al Estado significativas responsabilidades en la garantía de oportunidades. Esto puede ser más importante que la atención directa a los grupos menos favorecidos por medio de acciones redistributivas, mientras se confía ampliamente en la capacidad igualadora de buenas políticas de salud y educación (p. 127).

El rol del Estado como agente promotor o inhibidor de desigualdades sociales, es una manifestación del cambio de época. En el pasado -se dice- los recursos públicos favorecieron el acceso a las oportunidades y el intercambio entre las clases, en la actualidad contribuye al ensanchamiento de las distancias y a la fijación de las diferencias.

\section{Desigualdad y segmentación laboral}

Muchos autores han coincidido que el empleo es el eje motor del desarrollo de los países y que las políticas sociales deberían estar enfocadas a este componente. Si bien no es tarea de los Gobiernos crear empleo, las funciones gubernamentales son esenciales para el aumento sostenido de puestos de trabajo.

La calidad de la administración pública reviste importancia crucial para el desarrollo, se trate de maestros que enseñan conocimientos especializados, agentes de extensión agrícola que mejoran la productividad agropecuaria o planificadores urbanos que diseñan ciudades funcionales (Banco Mundial, 2013: s/p)

Si bien es cierto no es competencia del Estado la generación del empleo, 
pues esta acción es propia del sector privado, este debe garantizar que existan las condiciones para un firme crecimiento impulsado por el sector privado, donde se determine no solo de la cantidad de empleos necesarios para un mejoramiento de la distribución, si no también el tipo de empleos que se están propiciando al interior de la economía. En este sentido, algunos tipos de empleo son más beneficiosos para el desarrollo, mientras que otros tienen pocos efectos en él, aunque sean atractivos para las personas, como el caso de los empleos que son perjudiciales para el medio ambiente y que generan un alto costo que todos debemos pagar. Por tanto, son las circunstancias las que determinan cuál es el tipo de empleo más provechoso para el desarrollo, ya que los países difieren en sus niveles de desarrollo, su demografía, su riqueza natural y sus instituciones.

Así pues, las políticas sociales referentes al empleo deberían permitir al Estado, detectar cuáles empleos ofrecen el máximo beneficio para el desarrollo, según las circunstancias de cada país, estas deberían eliminar los obstáculos que impiden al sector privado crear el tipo de empleo que genera mayores ingresos y beneficios sociales. En síntesis la política laboral, dada la segmentación, debe facilitar la creación de empleo y mejorar sus efectos beneficiosos para el desarrollo.

El problema fundamental es que las pequeñas economías se caracterizan por agricultores y trabajadores por cuenta propia que están al margen del mercado de trabajo y especialmente carecen de técnicas agrícola, apropiadas que permitan maximizar y mejorar sus ingresos, inclusive estos empleos difieren entre países y al interior de los mismos, veamos el gráfico siguiente: (ver pagina 81 )

Obsérvese que el empleo en América Latina, se caracteriza por un elevando porcentaje (40\% aproximadamente) de empleo que no cobra salario, característica particular de sectores, educación y experiencia, la inexistencia de registro de las empresas o bien porque los empleados carecen de cobertura del sistema de seguridad social o contratos de trabajo.

Aunado a esto las disparidades por motivos de género y de edad son asombrosas. En todo el mundo tienen empleo menos de la mitad de las mujeres, en comparación con casi las cuatro quintas partes de los hombres, aunque son las mujeres las que continúan recibiendo ingresos significativamente menores

80 Derechos sociales y políticas sociales: en busca de la estabilidad y el equilibrio en América Latina

Fernando Montero-Cordero 


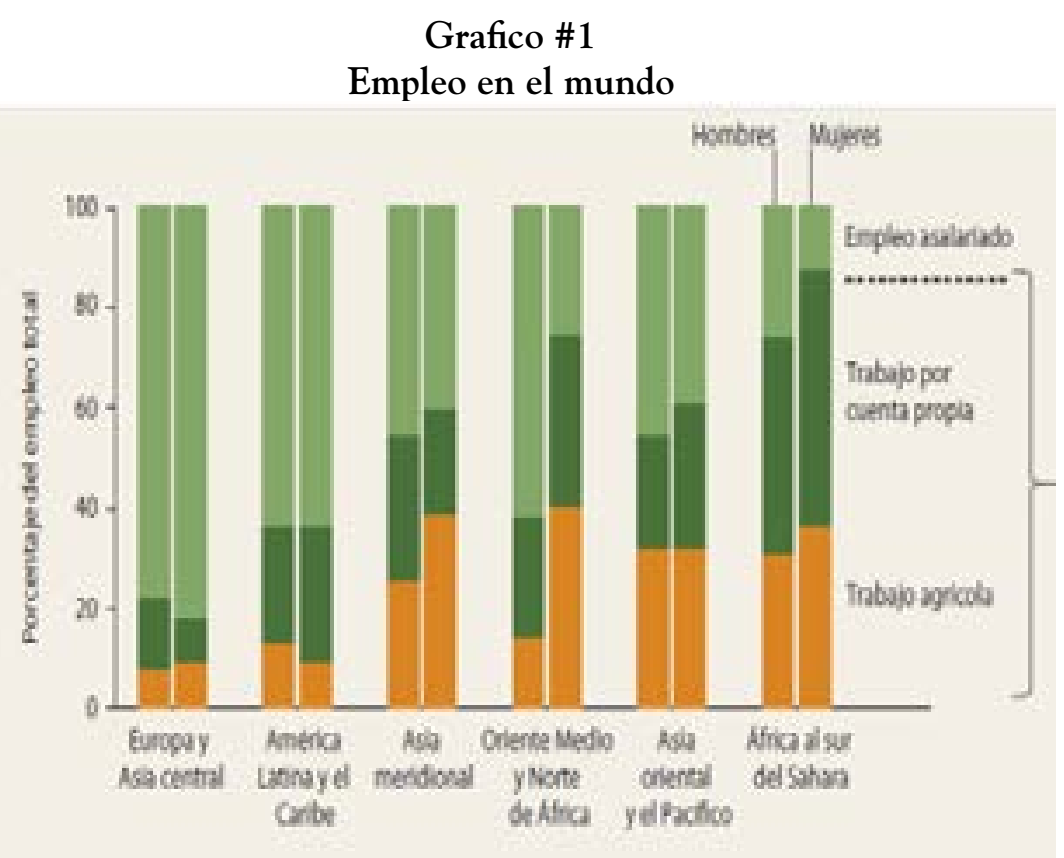

Fuente: Informe sobre el desarrollo humano, 2013, p. 5.

que los hombres. Otro elemento a considerar por las políticas sociales dentro del empleo es la rápida urbanización lo que está cambiando la composición del empleo. Producto de ello, la fuerza de trabajo agrícola será superada por la no agrícola y esto generará una necesidad mayor de empleos que requieran de especialización mediana, puesto que las tareas de producción se han fragmentado de tal forma que puedan realizarse en diferentes lugares.

Las grandes empresas transnacionales han canalizado esta segmentación por todo el mundo y esto ha traído consigo un reto enorme para los países en desarrollo, quienes deben valorar sus políticas laborales para insertarse sabiamente en dicha segmentación.

La exportación de servicios manufactureros de los países en desarrollo se ha duplicado en menos de una década, donde la tecnología y los sistemas de comunicación han sido fundamentales para la eficiencia de las empresa y por ende de los trabajadores. Este nuevo panorama de la producción global también ha 
producido cambios en las capacidades técnicas y en la distribución mundial de las personas con talentos especiales, son aquellos países que no han logrado transformar sus políticas laborales y de comercio lo que han relegado al sector productivo a procesos de valor cada vez menores.

Entonces, ¿es el empleo equitativo y justo el eje dinamizador y pilar esencial del desarrollo? Pues sí, el empleo puede elevar los niveles de vida, la productividad y el fomento de la cohesión social.

Muchas familias caen en la pobreza o escapan de ella porque sus miembros pierden u obtienen un empleo. Las oportunidades de empleo remunerado, incluso en la agricultura y el trabajo autónomo, ofrecen a los hogares los medios de aumentar el consumo y reducir su variabilidad. El aumento de los ingresos laborales repercuten sobre el desarrollo y el mejoramiento de la calidad de vida, pues a medida que el país se desarrolla el nivel medio de cualificación de quienes ejercen el empleo aumenta. Los estudios recientes revelan que los cambios en los ingresos laborales son el principal factor que reduce la pobreza y garantiza los derechos sociales.
Pero ¿cómo podemos mejorar los ingresos si el tipo de desempleo es estructural? Según Pérez Sainz, los desafíos en este aspecto van dirigidos a "acrecentar la empleabilidad de los jóvenes a través de una formación adecuada de capital humano y, en el segundo caso, con plantear la cuestión de la ciudadanía laboral a nivel centroamericano" (Pérez-Sáinz, 2010: p. 4).

Elementos como reducción del Estado, regulación y la generación de una nueva proletarización podrían ser claves en la acumulación y el desarrollo dentro de la desocupación estructural que viven las economías latinoamericanas. Las políticas de empleo por tanto deberán ser dirigidas según Pérez, en mantener los derechos laborales mínimos y apostar por las vías altas de la acumulación globalizada, hacia la maximización de oportunidades que plantea dicha globalización.

Otros autores, como Sojo (2010), señalan que la desigualdad laboral es legítima pero está gobernada por principios superiores de igualdad de derechos. Ello condiciona una autolimitación de los jefes, y una actitud reivindicadora de la condición laboral por parte de los empleados.

82 Derechos sociales y políticas sociales: en busca de la estabilidad y el equilibrio en América Latina 
Equilibrio hacia adentro...equilibrio hacia afuera

En las últimas dos décadas el desempeño macroeconómico de los países de América Latina y el Caribe mejoró significativamente desde el punto de vista de la estabilidad nominal, un objetivo de política que había resultado particularmente difícil de alcanzar en los años setenta y ochenta y principios de la década de 1990. Sin embargo, este logro —que suele asociarse con una tasa de inflación baja y estable y una situación fiscal equilibrada- no estuvo en general acompañado por un elevado y sostenido dinamismo económico, ni por una menor volatilidad de la actividad productiva. De hecho, el crecimiento económico de la región ha sido menor que el de otras economías emergentes. De acuerdo con la evidencia sobre los ciclos económicos presentada en el capítulo anterior, el ritmo de expansión económica de los países de América Latina y el Caribe experimentó grandes fluctuaciones en las últimas dos décadas, e incluyó serias crisis financieras y de balanza de pagos" (CEPAL, 2012: p. 161).

La estabilidad macroeconómica es, elemento fundamental para el desarrollo socioeconómico y uno de los pilares esenciales para la generación de políticas sociales asertivas, que aseguren los derechos ciudadanos y el mejoramiento de la calidad de vida. Pero la estabilidad no debe estar objetivizada exclusivamente en la reducción de la inflación y el equilibrio de las finanza públicas, debe también ser enfocada a los procesos productivos y a la suavización de los ciclos económicos, crecimiento económico con objetivos tendientes a una mejora en la distribución de los ingresos, tendientes a un cambio estructural favorable para el individuo y la comunidad. Es

...fundamental que la política macroeconómica dé un salto hacia adelante, adopte un enfoque que priorice de manera explícita el cambio estructural y nivele hacia arriba las capacidades y oportunidades sociales. De este modo disminuyen las brechas de productividad, lo que brinda sustento a la reducción efectiva de las desigualdades. Esto requiere una concepción de la estabilidad que, más allá del control de la inflación, sea funcional para el desarrollo y que la visión excesivamente compartimentada de la micro y la macroeconomía sea sustituida por un enfoque integrado que preste adecuada atención a las interrelaciones entre ambas (CEPAL, 2012: p. 163). 
La política macroeconómica puede influenciar en la estructura productiva, afectando sectores productivos, flujos de inversión, la política fiscal regula la actividad económica a través del gasto y la inversión pública y social; la política monetaria influye sobre los macroprecios, la balanza de pagos, tasas de interés, tipos de cambio y el comercio de bienes y servicios en general.

La política fiscal no solo se ha transformado en un factor estabilizador de las expectativas de los agentes económicos sino que además se le ha otorgado un papel más protagónico en la creación de empleo y el sostenimiento del dinamismo económico de América Latina

El crecer para igualar e igualar para crecer, es un concepto esencial, pues no se contraponen, todo lo contrario, presentan una independencia que se retroalimenta y permite alcanzar una mayor y mejor igualdad y repartición de la renta nacional.

Parte de esa equidad es garantizar los recursos necesarios para la aplicación de políticas sociales a través del los recursos públicos y el gasto social, pero el cuestionamiento viene respecto a cuánto del crecimiento de los recursos fiscales van dirigidos al gasto público. ¿Cuál es el margen de endeudamiento de un Estado que permita invertir parte de sus recursos económicos en la sociedad sin comprometer sus finanzas? Es interesante como países productores de hidrocarburos y minerales mantuvieron gastos sociales por debajo del crecimiento de sus ingresos en época de crisis y el resto de países de América Latina y el Caribe, mantuvieron estables y hasta incrementaron parte de su gasto, permitiendo controlar la crisis y por ende la afectación de la desigualdad.

El gasto público social en América Latina ha registrado una tendencia creciente, independientemente de la trayectoria de los pagos de intereses de la deuda pública, aumentando del 44,9\% del gasto público total en el período comprendido entre 1990 y 1991 al 62,2\% en el período comprendido entre 2008 y 2009.

La política monetaria tiene un papel importante en la búsqueda de la estabilidad y el crecimiento, actuando de forma coordinada con la política fiscal. La combinación de programas de estabilización basados en el tipo de cambio como ancla nominal y reformas de mercado, particularmente apertura comercial, dio lugar a un fuerte alineamiento de los precios de los bienes y servicios transables con los precios externos, que coadyuvó

84 Derechos sociales y políticas sociales: en busca de la estabilidad y el equilibrio en América Latina

Fernando Montero-Cordero 
al logro de la estabilización en los países Latinoamericanos.

La apertura comercial y la sustitución de oferta nacional por oferta importada, la apreciación de las monedas locales y la caída de los precios internacionales de los hidrocarburos y los metales y minerales contribuyeron a reducir las presiones inflacionarias por el lado de la oferta, al disminuir los costos laborales y de los insumos y bienes finales transables, impactando positivamente en el consumo y en el mejor manejo de los ingresos en todos los sectores de la economía.

Para garantizar la protección social, las economías tienen un gran desafío macroeconómico, que es impulsar actividades de alta productividad, lo cual constituye una política social altamente distributiva, esto con el fin de romper la heterogeneidad estructural de las economías. Un conjunto de políticas sociales y desarrollo tendientes al cambio estructural necesario para poseer sectores de alta productividad, es fundamental en la generación de mejores y mayores ingresos que permitan romper con las "deficiencias históricas" en materia de protección social, vulnerabilidad de las crisis y la afectación del mercado de trabajo.

\section{Consideraciones a modo de cierre}

La desigualdad en América Latina viene dada por el funcionamiento interactivo de una cadena compuesta por la heterogeneidad estructural, el mercado de trabajo y la protección social. Es por tanto el punto de partida de cualquier política social combatir dicha heterogeneidad a partir del cambio estructural y romper con la reproducción sistemática de la desigualdad.

La gestión gerencial del Estado se convierte en el mecanismo idóneo para dirigir eficaz y efectivamente la institución pública, alcanzar los objetivos, horizontalizar las acciones sociales y sepultar cualquier condición de proteccionismo, de falsa inclusión y desmejoramiento de las condiciones de igualdad, principalmente en la aplicación de un Estado de derecho que posibilite transparentemente ejercer su acción igualitaria. Hablamos de una política pública que impulsa el desarrollo y corrige las inequidades que permitan el derecho a la salud, organización indígena, educación, ciudadanía, trabajo, etc. Una construcción de políticas inclusivas que integren al ciudadano de "a pie".

El trabajo es el motor principal de la inclusión social porque permite unir 
el desarrollo con carácter productivo $\mathrm{y}$ de alto nivel con la igualdad social y riqueza social. La definición de una política social debe estar enfocada en elementos como la desigualdad social, mercados laborales altamente diferenciados y con distintos niveles de ocupación e ingresos. Por ello reviste especial interés las acciones que el Estado genere para brindar mejores oportunidades de retribuir las capacidades productivamente, así como condiciones superiores para armonizar los intereses de los distintos actores del ámbito laboral.

No es solo la cantidad idónea de trabajo la que permite "igualar" y garantizar los derechos sociales, es la profundización en la búsqueda de empleos y opciones de ocupación de alta calidad que generen mayores beneficios para el desarrollo. En un mundo laboral altamente segmentado, los Estados deben propiciar aquellas políticas de fomento a la productividad y el empleo, que posibiliten al sector privado enlazar ocupaciones de alto valor agregado, principalmente aquellas que sean relaciones necesarias y de largo plazo. El caso particular de Costa Rica, un país con desigualdades múltiples, hacer eficiente la inversión en el logro de salarios mínimos, con ligamen al desarrollo local y donde la gente se inserte a nuevas formas de empleo.
Del lado de la estabilidad económica, las políticas fiscales contractivas significan un gran desafío para las economías en desarrollo, buscando espacios fiscales a través de la combinación de un buen uso, es decir, eficiente, del gasto que asegure un impulso a la demanda agregada y por otro lado, que generen el crecimiento de las economías en la fase contractiva de los ciclos. Las políticas macroprudenciales permiten que los espacios fiscales generados en épocas de auge permitirían a las economías un ahorro público que permita suavizar los embates de choques adversos sin poner en peligro la sostenibilidad financiera de la economía.

Para la gestación de políticas de protección, es fundamental tener claro quiénes son los actores sociales, quienes reciben los servicios o quiénes son los beneficiados. Es necesario crear indicadores de gestión pública que permitan valorar el impacto real de dicha políticas.

Por último la política social debería ser adecuada, integral, profunda, sostenible económicamente en el tiempo, de cierta calidad y que promueva el desarrollo humano, la integralidad y el mejoramiento continuo de la calidad de vida para todos los ciudadanos, sin distinción de clase, estrato, o condición socioeconómica.

86 Derechos sociales y políticas sociales: en busca de la estabilidad y el equilibrio en América Latina Fernando Montero-Cordero 


\section{Referencias bibliográficas}

CEPAL. (2012). Cambio estructural para la igualdad. Santiago: Naciones Unidas.

Banco Mundial. (2013). Informe sobre el desarrollo mundial, 2013. Empleo. Panorama General. Washington D. C: Banco Internacional de Reconstrucción y Fomento/Banco Mundial. Recuperable en: siteresources. worldbank.org/EXTNWDR2013/ Resources

O'Donnell, G. (2001). La irrenunciabilidad del Estado de Derecho. Barcelona: Institut Internacional de Governabilitat de Catalunya, Córsega.

Pérez-Sáinz, J. P. (2010). Las cuentas pendientes de la modernización. Tendencias laborales y sus efectos sobre la integración social en el Istmo Centroamericano. EE.UU: Oficina de Desarrollo Regional y Sostenible, América Latina y el Caribe (LAC). Recuperable de: CA2020.fiu.edu/Juan_Pablo/JPPS.pdf

Sojo, C. (2010). Igualiticos la construccion social de la desigualdad en Costa Rica. San Jose: PNUD.

Vargas, A. F. (10 de 10 de 2012). La gerencia social y el nuevo paradigma de gerencia. Revisado el 10/10/2013. Recuperable de http://www.ts.ucr.ac.cr/binarios/ docente/pd-000170.pdf 